\title{
Design of a Moving-magnet Electromagnetic Actuator for Fast Steering Mirror through Finite Element Simulation Method
}

\author{
Yongjun Long, Jinqiu Mo, Xiaohui Wei, Chunlei Wang, and Shigang Wang* \\ The State Key Laboratory of Mechanical System and Vibration, School of Mechanical Engineering, \\ Shanghai Jiao Tong University, 800 Dongchuan Road, Shanghai 200240, China
}

(Received 3 June 2014, Received in final form 17 July 2014, Accepted 24 July 2014)

\begin{abstract}
This paper develops a moving-magnet electromagnetic actuator for fast steering mirror (FSM). The actuator achieves a reasonable compromise between voice coil actuator and piezoelectric actuator. The stroke of the actuator is between the strokes of a piezoelectric actuator and a voice coil actuator, and its force output is a linear function of air gap and excitation current within our FSM travel range. Additionally, the actuator is more reliable than voice coil actuator as the electrical connection in the actuator is static. Analytically modeling the actuator is difficult and time-consuming. Alternatively, numerous finite element simulations are carried out for the actuator analysis and design. According to the design results, a real prototype of the actuator is fabricated. An experimental test system is then built. Using the test system, the force output of the fabricated actuator is evaluated. The test results validate the actuator analysis and design.
\end{abstract}

Keywords : fast steering mirror, electromagnetic actuator, moving-magnet, finite element simulation

\section{Introduction}

Fast steering mirror (FSM) is a beam-adjusting device that is widely used in adaptive optical system such as laser communication, imaging system and target-tracking laser radar. As a key component of adaptive optical system, FSM utilizes a reflective mirror to reflect a beam to a target position with high accuracy and high response speed, and to reject potential disturbances to achieve very small beam tilting errors. To achieve fast and accurate beam pointing or tracking, FSM must have sufficient accuracy and bandwidth. Besides, FSM should have enough scanning area to provide adequate pointing between two long distance terminals. For some special applications such as laser communication between satellites, FSMs with compact structures and low power consumptions are required.

The performances of an FSM, such as bandwidth, scanning range, and power consumption are affected by its actuators significantly. Generally, FSMs are driven by voice coil actuators [1-5] or piezoelectric actuators [6-10]. Voice coil actuator is based on Lorentz force and allows

(C)The Korean Magnetics Society. All rights reserved.

*Corresponding author: Tel: +86-21-34204496

Fax: +86-21-34204496, e-mail: wangshigang@sjtu.edu.cn long actuation stroke with little power input. However, the maximum force output of voice coil actuator is limited as the maximum current carrying capacity of the conductor used in the coil windings is restricted by the coil overheat problem. Conversely, piezoelectric actuator offers relatively high acceleration but accompanied by a short stroke which is caused by its small proportional coefficient of strain to applied voltage and by its inherent numerous electrical and mechanical losses when operating in high frequency. Therefore, the FSMs driven by voice coil actuators allow wider scanning areas but lower bandwidths whereas the FSMs using piezoelectric actuators have higher bandwidths but smaller scanning areas. The ideal alternative to FSM actuator should combine the advantages of both voice coil actuator and piezoelectric actuator. Bandera [11] achieved a fast steering mirror by using an electromagnetic actuator which accomplishes a reasonable compromise between voice coil actuator and piezoelectric actuator. However the detailed analysis and design of the electromagnetic actuator were not included and the way of solving the actuator's nonlinear problem was not addressed in Ref. [11]. Kluk et al. designed two fast steering mirrors named the AFSM (Advanced Fast Steering Mirror) and sAFSM [12] (small Advanced Fast Steering Mirror) by using flux-biased electromagnetic actuators $[13,14]$. The AFSM and SAFSM actuators have 
several advantages, such as high force densities, linear torque outputs and reasonable effective strokes. However, the actuators have high inductances which have significant effects on the design of the power amplifiers driving the actuators. To achieve high bandwidths of the AFSM and sAFSM, amplifiers with high power outputs are required due to the high inductances. Besides, the electromagnetic structures of the AFSM and SAFSM actuators are relatively complex than that of Bandera's FSM actuator.

This paper is motivated by the detailed modeling, analysis and design of a moving-magnet electromagnetic actuator, which compromises between voice coil actuator and piezoelectric actuator, for fast steering mirror through a series of finite element simulations which are carried out by Ansoft Maxwell 15.0. Based on the simulation results, the effects of the major design parameters on the actuator's force output are analyzed and the way of determining these major design parameters is introduced in detail. Both the simulation and experimental results show that the actuator's force output is nearly a linear function of the driving current and actuating displacement. Driven by the actuator, a compact FSM with bandwidth on the order of hundreds of hertz and optical scanning range on the order of tens of milliradians is expected to be achieved.

\section{Structure and Operating Principle Description}

To achieve an FSM with good performances, a movingmagnet electromagnetic actuator is designed to drive the FSM. In this section, the structures and operating principles of the FSM and its actuator are presented in an intuitive way.

\subsection{Structure Description}

Figure 1 shows the exploded view and a real fabricated prototype of our FSM. Driven by four electromagnetic actuators each of which consists of a permanent magnet, a magnet holder, a coil core, an excitation coil winding and a flux directing steel, the FSM is able to generate two rotary motions, $\theta_{X}$ and $\theta_{Y}$, simultaneously. To present the structure and operation clearly, the FSM is divided into three modules named the mirror module, movable module and static module.

The mirror module consists of the mirror, mirror base and mirror mounting piece. It uses the reflective mirror to reflect a beam to a target position. The mirror module is the controlled object and the mirror steering angle is the output of the FSM system.

Along with the mirror module, the movable module is a movable part of the FSM. The movable parts of the FSM,

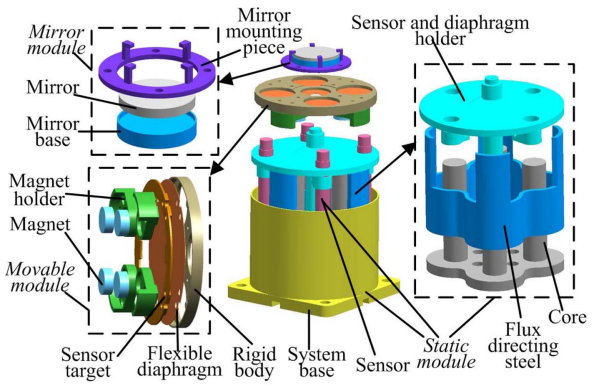

(a)

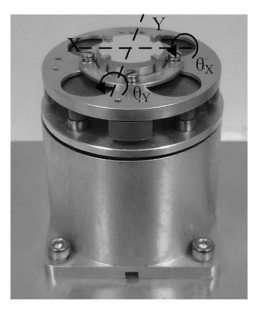

(b)
Fig. 1. (Color online) Exploded view and a real fabricated prototype of the FSM. (a) Exploded view, (b) a real fabricated prototype.

including the mirror module and movable module, are suspended by the flexible diaphragm which is used to generate restoring torque against actuating torque to yield angle control.

The static module is the immovable part of the FSM. The movable module is mounted to the static module though the flexible diaphragm and diaphragm holder. Four non-contacting displacement sensors and the sensor target in the movable module make up the angle detection system which is used to detect the steering angle of the mirror in real time to yield steering angle closed-loop control. Hence, a high pointing accuracy of the FSM can be achieved. The core is used to wind exaction coil windings and conduct magnetic flux. For convenience of fabrication and assembling, both the cores and flux directing steels of the four actuators are respectively made to be a whole.

\subsection{Operating Principle}

Figure 2 shows the schematic cross-sectional view of the FSM [11]. Four moving-magnets and their holders are suspended by the flexible diaphragm while four corresponding cores, four corresponding coil windings and four corresponding flux directing steels are fixed to the static system base. The moving-magnets, cores, coil windings and flux directing steels make up four independent electromagnetic circuits. In each circuit, an attraction force is generated between the moving part and static part.

The attraction force in each magnetic circuit is generated by the magnetic field $B$ between the moving part and static part. The magnetic field $B$ is the superposition of the magnetic field $B_{P M}$ generated by the permanent magnet and the magnetic field $B_{\text {Coil }}$ generated by the coil. Hence, the electromagnetic attraction force $F_{e}$ can be expressed as

$$
F_{e}=f\left(B_{P M}, B_{C o i l}\right)
$$




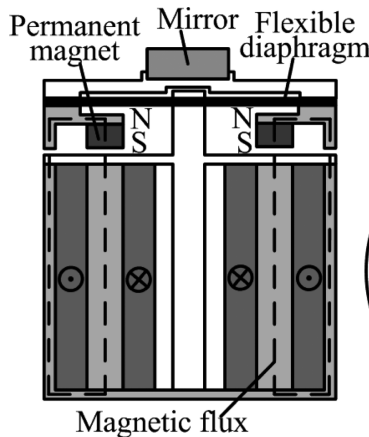

(a)

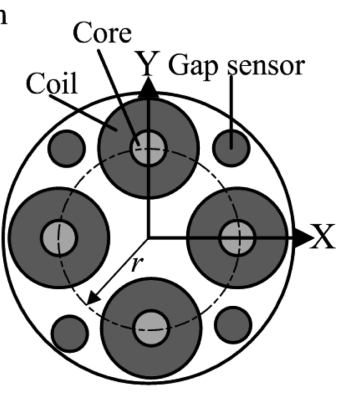

(b)
Fig. 2. Schematic cross-sectional view of the FSM. (a) Side view, (b) top view.

At initial state, the coil in each circuit is not excited. Therefore, the magnetic field $B$ is equal to the magnetic field $B_{P M}$. Due to the symmetry of the FSM, the magnetic field $B_{P M}$ in each magnetic circuit is identical. Hence, the attraction forces in the magnetic circuits are equal to each other at initial state. That is,

$$
F_{e}^{-X}=F_{e}^{+X}=F_{e}^{-Y}=F_{e}^{+Y}=F=f\left(B_{P M}, 0\right)
$$

where $F_{e}^{-X}$ indicates the attraction force of the " $-X$ " actuator, $F_{e}^{+X}$ indicates the attraction force of the " $+\mathrm{X}$ " actuator, and so on.

The actuating torque of each axis can be given by

$$
\left\{\begin{array}{l}
T_{X}=T_{X 1}-T_{X 2}+2 F_{e}^{-Y} r-2 F_{e}^{+Y} r \\
T_{Y}=T_{Y 1}-T_{Y 2}+2 F_{e}^{-X} r-2 F_{e}^{+X} r
\end{array}\right.
$$

where $T_{X}$ is the actuating torque pointing to the positive $\mathrm{X}$-direction and $T_{Y}$ is the actuating torque pointing to the positive Y-direction.

Combing Eq. (2) and Eq. (3), we can obtain that $T_{X}=$ $T_{Y}=0$. Hence, the torque acting on the flexible diaphragm is null at the initial state and no steering angle is produced. Figure 3(a) shows the flexible diaphragm deformation when setting the excitation currents of the four coils at zero. Although the torque acting on the flexible diaphragm is null, the resultant force acting on the flexible diaphragm is not null. Hence, the FSM has a constant vertical displacement respect to the natural state of the flexible diaphragm.

If the two coils in the X-direction are energized as Fig. 2(a) shows, two additional magnetic fields, $B_{\text {Coil }}^{-X}$ and $B_{\text {Coil }}^{+X}$, are generated in the " $-\mathrm{X}$ " and " $+\mathrm{X}$ " circuits, respectively. As the directions of $B_{C o i l}^{-X}$ and $B_{P M}$ are identical, the magnetic field of the " $X$ " circuit is enhanced. Simultaneously, the magnetic field of the " $X$ " circuit is reduced because the directions of $B_{C o i l}^{+X}$ and $B_{P M}$ are

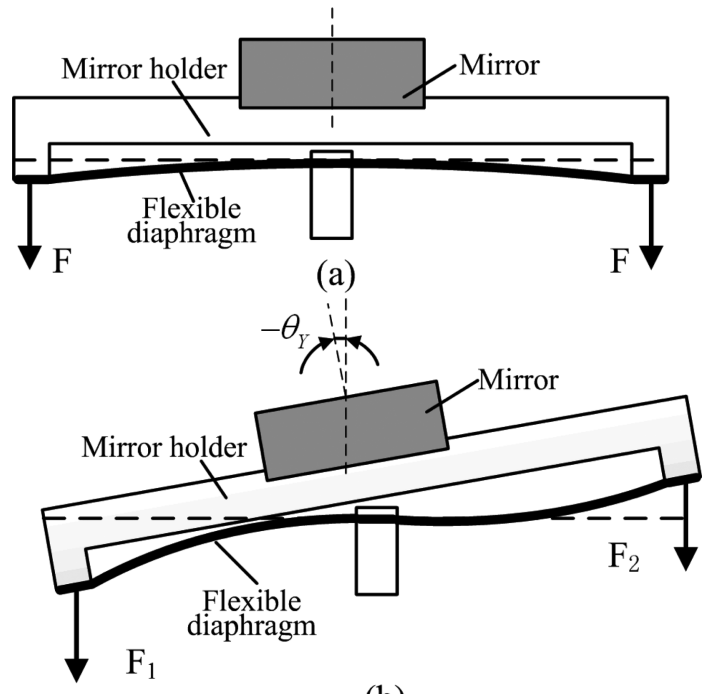

(b)

Fig. 3. Flexible diaphragm deformation. (a) At the initial state, (b) when excitation currents are assigned.

opposite. As we know, electromagnetic force increases with magnetic field for the actuator used in our FSM. Hence, $F_{e}^{-X}$ increases form $F$ to $F_{1}\left(F_{1}>F\right)$ while $F_{e}^{+X}$ decreases from $F$ to $F_{2}\left(F_{2}<F\right)$ after the coils are energized. Consequently, a torque $T_{Y}$ pointing the negative Ydirection is generated on the flexible diaphragm.

$$
T_{Y}=2 F_{e}^{+X} r-2 F_{e}^{-X} r=2 r\left(F_{2}-F_{1}\right)<0
$$

Driven by $T_{Y}$, a steering angle $-\theta_{Y}$ is then produced. The generation of the steering motion $\theta_{X}$ is the same as that of $\theta_{Y}$. Figure 3(b) shows the flexible diaphragm deformation when the two coils in the X-direction are energized as Fig. 2(a) shows. As $F_{2}+F_{1} \approx 2 F$, the vertical displacement respect to the natural state of the flexible diaphragm almost keeps constant.

Note that the direction of the magnetic field $B$ is always the same as that of the magnetic field $B_{P M}$ generated by the permanent magnet. That is, the coil magnetic field $B_{\text {coil }}$ which is opposite to $B_{P M}$ should not exceed $B_{P M}$. In fact, generating a coil magnetic field $B_{\text {coil }}$ that exceeds $B_{P M}$ needs a large current. Therefore, we design that $B_{\text {Coil }}$ $<B_{P M}$ to decrease power requirement and improve energy efficiency.

Voice coil actuator generates actuation as a result of Lorentz forces on moving coils. The moving-magnet actuator in this paper produces actuation based on the variation of the magnetic field between the static part and moving part. Compared with voice coil actuator, the moving-magnet actuator is much simpler and easier to fabricate. Besides, moving-magnet type actuator is more reliable than moving-coil type actuator as the electrical 
connection in moving-magnet type actuator is static. Since the proportional coefficient of force to applied current decreases with air gap length greatly, the effective stroke of the moving-magnet actuator is smaller than that of a voice coil actuator. However, it is much larger than that of a piezoelectric actuator. With the previous analysis, it can be concluded that the moving-magnet actuator in this paper achieves a reasonable balance between voice coil actuator and piezoelectric actuator.

\section{Modeling and Design via Finite Element Simulation}

Theoretically, it is far more convenient for us to design the electromagnetic actuator if we can obtain the analytical model of the actuator. However, the electromagnetic analysis of the actuator is complex and analytically modeling the actuator is a difficult and time-consuming work. Alternatively, this paper utilizes numerous finite element simulations done on Ansoft Maxwell 15.0 to analyze, model and design the actuator. Making use of a computer with high performances, these simulations can be accomplished without too much time cost. Especially, these simulations can be carried out automatically without human intervention by creating a parametric simulation model and by using Ansoft Maxwell's parametric simulation function. Based on the simulation results, the effects of the major design parameters on the actuator's force output are analyzed and the actuator design is presented in detail.

\subsection{Simulation Model}

For convenience of fabrication and assembling, both the cores and flux directing steels of the actuators in the FSM are respectively made to be a whole. Theoretically, the 3D magnetic simulation model should be that shown by Fig. 4(a). However, the simulation model can be simplified as Fig. 4(b) shows because of the electromagnetic independence between each actuator (it will be proved in the following analysis). That is, it is capable of including only one actuator in the simplified simulation model. As a result, the simulation time cost decreases significantly.

\subsection{Simulation Conditions}

To simplify the simulations, some design parameters of the actuator have been determined in advance. The maximum required coil magnetomotive force is restricted by the power amplifier used to drive the actuator. According to the output capability and frequency response of the power amplifier that we have, the coil turn and maximum excitation current are determined to be 70 turns and $3 \mathrm{~A}$,

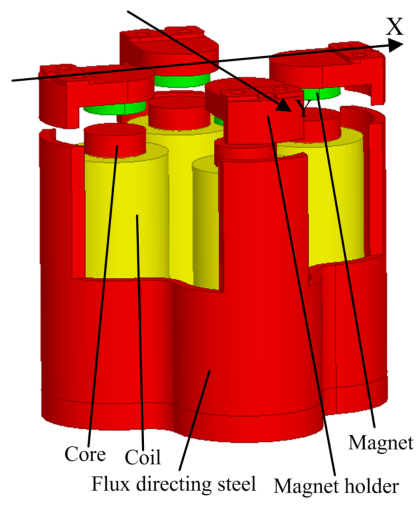

(a)

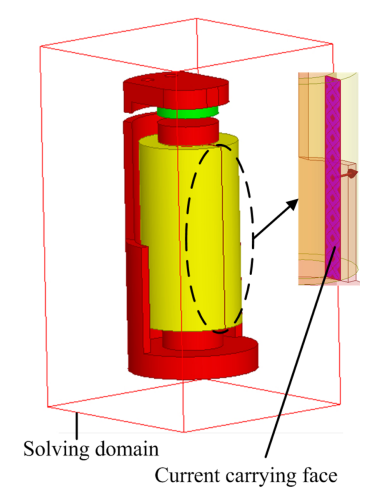

(b)
Fig. 4. (Color online) Electromagnetic finite element simulation model. (a) Initial complete model, (b) simplified model with only one actuator.

respectively. The core length should be large enough to wind a coil of 70 turns and is determined to be $29.5 \mathrm{~mm}$. To prevent eddy current loss and overheat problem, the ideal core should be laminated with material of high permeability such as nickel-iron alloy. But for quick concept verification, the cores of the first FSM prototype are fabricated with magnetic stainless steel (2Cr13). The cores made of magnetic stainless steel have high eddy current loss, but they are very easy to manufacture. Hence, they are ideal for quick concept verification. Along with the cores, the magnet holders and flux directing steels are also made of $2 \mathrm{Cr} 13$ stainless steel. The remanence of the permanent magnet (neodymium-iron-boron) is $1.2 \mathrm{~T}$. The current carrying face is used to assign excitation current source to the coil. The excitation current enhancing the magnetic field of the actuator is defined to be positive. A cubic with 'vacuum' material assigned is created to limit the solving domain of the simulations.

Ansoft Maxwell can mesh the model automatically. It refines the mesh iteratively until the output variable converges sufficiently. In order to achieve high computation accuracy, the initial mesh of the simulation model is achieved manually in this paper. Especially, a fine mesh is assigned to the top face of the core and the bottom face of the magnet. Figure 5 shows the initial mesh.

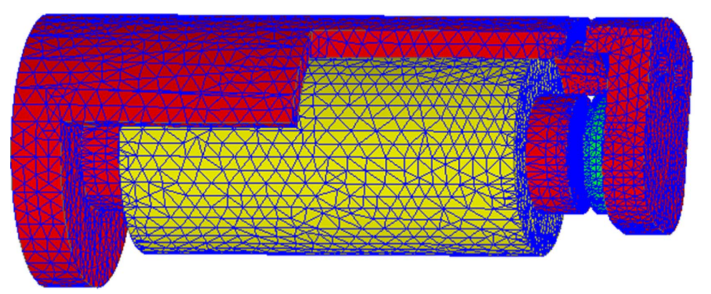

Fig. 5. (Color online) Initial mesh of the simulation model. 


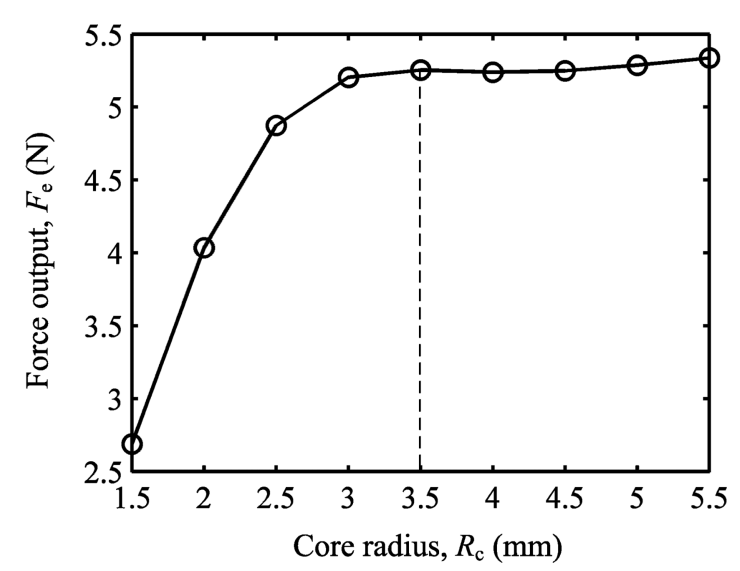

Fig. 6. Variation of the force output with the core radius.

Figure 6 shows the variation of the force output $F_{\mathrm{e}}$ with the core radius $R_{\mathrm{c}}$ when the magnet radius keeps constant. The simulation results shown in Fig. 6 are obtained by setting $R_{\mathrm{m}}=3.5 \mathrm{~mm}, H_{\mathrm{m}}=2.5 \mathrm{~mm}, L_{\mathrm{g}}=1 \mathrm{~mm}$ and $I=3$ A where $R_{\mathrm{m}}, H_{\mathrm{m}}, L_{\mathrm{g}}$ and $I$ are the magnet radius, magnet height, air gap length and excitation current, respectively. From Fig. 6, it can be seen that the actuator's force output $F_{\mathrm{e}}$ is mainly determined by the smaller one between the core radius and magnet radius if the other design parameters keep constant. That is, increasing the larger radius will not affect the force output significantly. With this feature taken into account, the core radius is kept equal to the magnet radius. Therefore, the design parameters varying in the simulations include the magnet radius $R_{\mathrm{m}}$, magnet height $H_{\mathrm{m}}$, and air gap length $L_{\mathrm{g}}$. Setting the magnet radius $R_{\mathrm{m}}$, magnet height $H_{\mathrm{m}}$, air gap length $L_{\mathrm{g}}$ and excitation current $I$ to variables and adding them to the simulation model, we can conveniently obtain the actuator's force output $F_{\mathrm{e}}$ as a function of the magnet radius $R_{\mathrm{m}}$, magnet height $H_{\mathrm{m}}$, air gap length $L_{\mathrm{g}}$ and excitation current $I$ by using the parametric solution of Ansoft Maxwell.
The variable ranges for the parametric solution are specified as follows:

$$
\begin{gathered}
1 \mathrm{~mm} \leq H_{\mathrm{m}} \leq 4 \mathrm{~mm} \\
2.5 \mathrm{~mm} \leq R_{\mathrm{m}} \leq 4.5 \mathrm{~mm} \\
0.5 \mathrm{~mm} \leq L_{g} \leq 3 \mathrm{~mm} \\
-3 \mathrm{~A} \leq I \leq 3 \mathrm{~A}
\end{gathered}
$$

\subsection{Design of the Actuator Parameters}

Based on the simulation model created in Sec. 3.1 and Sec. 3.2, numerous simulations with varying magnet radius $R_{\mathrm{m}}$, varying magnet height $H_{\mathrm{m}}$, varying air gap length $L_{\mathrm{g}}$ and varying excitation current $I$ are carried out. By using the simulation results, the methods of determining $R_{\mathrm{m}}, H_{\mathrm{m}}$ and $L_{\mathrm{g}}$ are introduced in detail.

\subsubsection{Magnet Radius and Magnet Height}

To achieve high dynamic stiffness, a maximum force output of the actuator is preferable. In addition, increasing the force output makes it possible to design a flexible diaphragm with high strength. As a result, the fatigue life of the flexible diaphragm is improved, therefore improving the life of the FSM.

Figure 7 shows the effects of the magnet height and radius on the actuator's force output when $L_{\mathrm{g}}=2 \mathrm{~mm}$ and $I=3 \mathrm{~A}$. It can be seen that the force output is an increasing function of the magnet height and radius. Compared with the magnet height, the magnet radius has a more remarkable effect on the force output. From the perspective of increasing the force output, a magnet with large height and large radius is preferable. However, increasing the height and radius of the magnet may lead to a magnet of great mass, therefore increasing the moment of inertia about the steering axis. As a result, the acceleration and bandwidth of the FSM may reduce. From the perspective

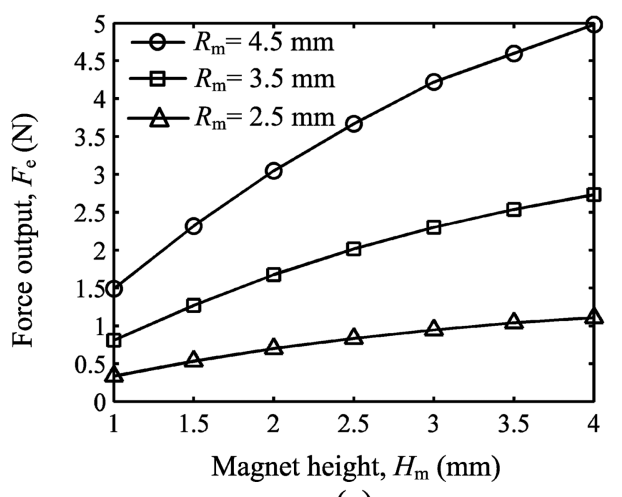

(a)

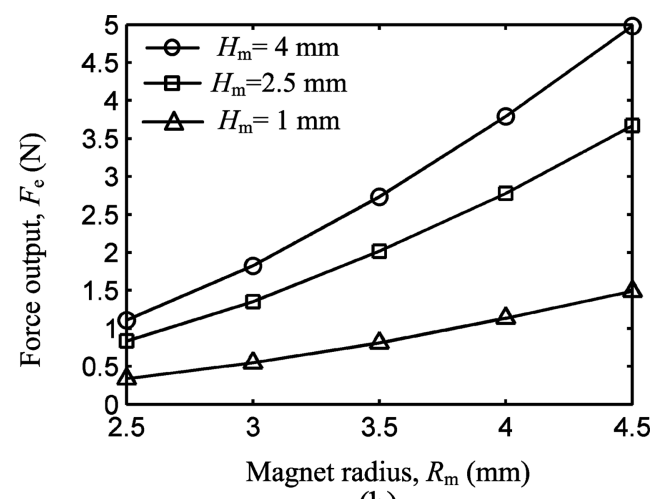

(b)

Fig. 7. Effects of the magnet height and magnet radius on the force output. (a) Effect of the magnet height, (b) effect of the magnet radius. 
of improving dynamic performance, a magnet with too large height and too large radius is not allowed.

The magnet height and radius should achieve a good balance between the force output and dynamic performance. Besides, designing the magnet is restricted by the size constraint of the FSM. Considering these limitations, the magnet height and magnet radius are specified at 2.5 $\mathrm{mm}$ and $3.5 \mathrm{~mm}$, respectively.

\subsubsection{Initial Air Gap Length}

The initial air gap length is a key design parameter. It determines the variation range of the air gap length which changes in real time when the FSM works. The initial air gap length has a significant effect on the actuator's force output so as to affect the performances of the FSM greatly. Hence, a detailed design of the initial air gap length is required.

Figure 8 plots the simulation force output versus the air

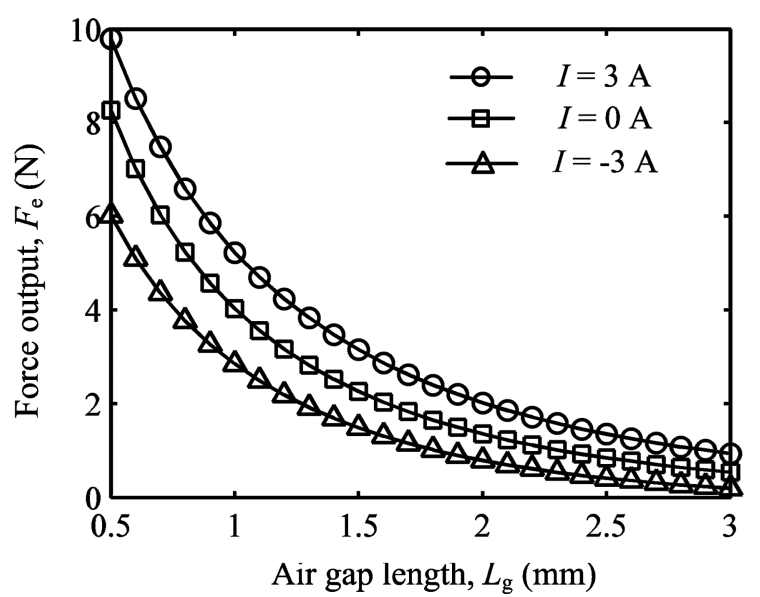

Fig. 8. Variation of the force output with the air gap length $\left(R_{\mathrm{m}}=3.5 \mathrm{~mm}\right.$ and $\left.H_{\mathrm{m}}=2.5 \mathrm{~mm}\right)$.

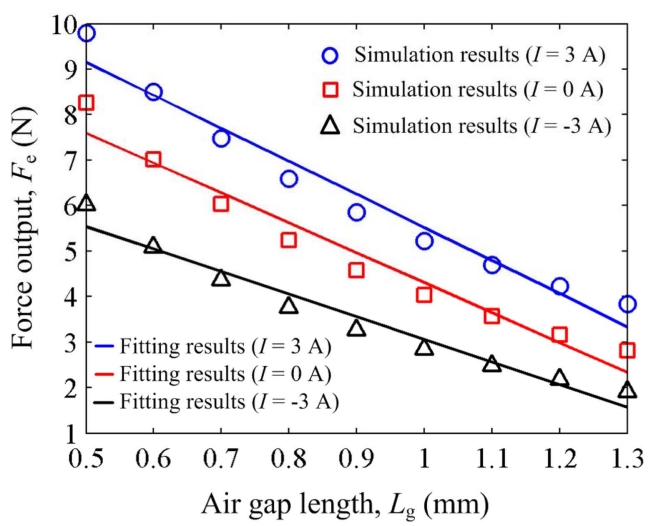

(a) gap length $\left(R_{\mathrm{m}}=3.5 \mathrm{~mm}\right.$ and $\left.H_{\mathrm{m}}=2.5 \mathrm{~mm}\right)$. It can be seen that the attraction force output decreases with the air gap length significantly. The force output is a nonlinear function of the air gap length and shows an inversely quadratic dependency on the air gap length. The effective rotation radius of the magnet, $r$ [refer to Fig. 2(b)], has been specified to be $11.5 \mathrm{~mm}$ according to the technical requirements of the FSM and the performances of the gap sensors. As the FSM has a travel range of $\pm 35 \mathrm{mrad}$, the travel range of the magnet is obtained to be $\pm 0.4 \mathrm{~mm}$. Figure 9 shows the relationship between the simulation force output and air gap length when the variation range of the air gap length is limited in the magnet travel range, along with the force output obtained from linear curve fitting. With a detailed analysis on Fig. 9, one can find that although the force output has a nonlinear dependency on the air gap length, it is nearly a linear function within the magnet travel range. The difference between Fig. 9(a) and Fig. 9(b) illustrates that the linear characteristic is more obvious when the air gap length has a larger value. From the perspective of improving linearity, an air gap with large length is preferable.

The force output as a function of the excitation current in the coil is shown in Fig. 10. The good agreement between the simulation results and linear fitting results confirms that the force output varies with the excitation current linearly. The slope of the linear curve of the force output versus the excitation current is not a constant and varies with the air gap length. The slope of the force output curve as a function of the air gap length is shown in Fig. 11. The slope of the force output curve, which denotes the gain from the excitation current to the force output, decreases with the air gap length significantly. Small slop of the force output curve indicates low energy efficiency. From the perspective of maintaining high energy

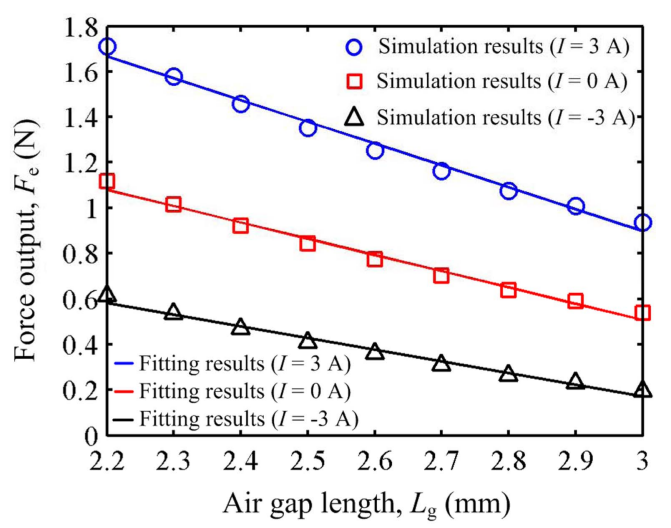

(b)

Fig. 9. (Color online) Simulation force output within the magnet travel range. (a) Air gap length with smaller value, (b) air gap length with larger value. 


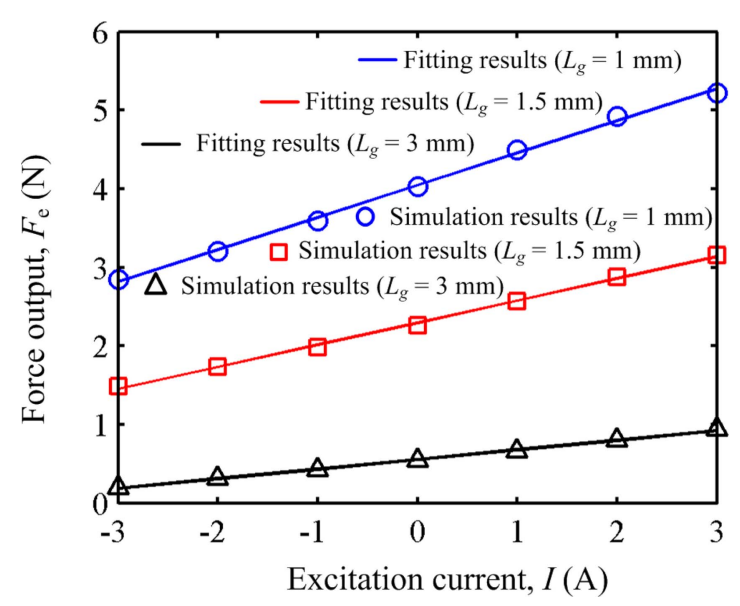

Fig. 10. (Color online) Simulation force output as a function of the excitation current.

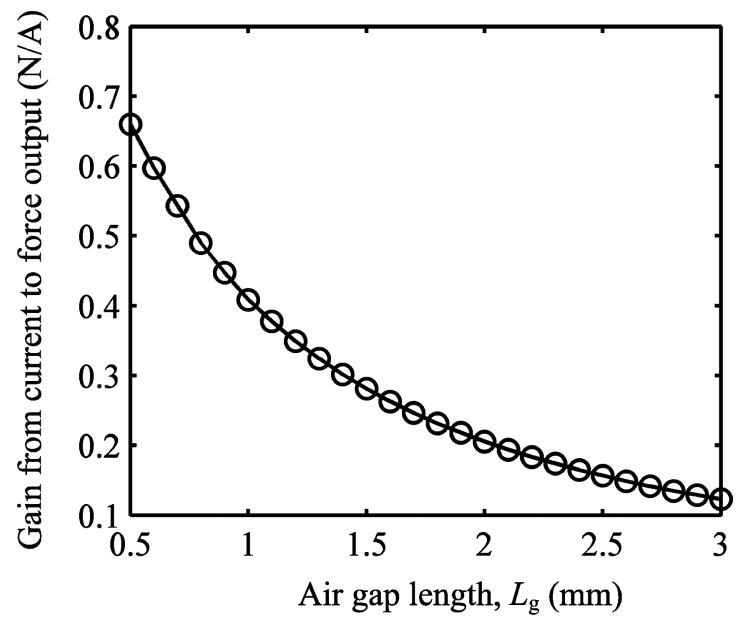

Fig. 11. Variation of the gain from the current to the force output with the air gap length.

efficiency, an air gap with small length is preferable.

Considering the requirements of linearity and energy efficiency, the initial air gap length is specified at $2 \mathrm{~mm}$ to compromise between linearity and energy efficiency. That is, the air gap length ranges from $1.6 \mathrm{~mm}$ to $2.4 \mathrm{~mm}$ when the FSM works. Figure 14 and Fig. 15 respectively plot the simulation force output versus the air gap length and the excitation current when setting $1.6 \mathrm{~mm} \leq L_{\mathrm{g}} \leq 2.4$ $\mathrm{mm}$, along with the linear fitting results.

\subsection{Electromagnetic Independence Validation}

In the previous analysis, the finite element simulations are carried out by using a simplified model which is created under the assumption that the actuators in the FSM are electromagnetically independent between each other. In order to validate the assumption, several simulations are implemented on the initial complete model [refer to Fig. 4(a)] which is created based on the actuator designed in Sec. 3.3. In the complete model, the excitation currents assigned to the coils positioned at 180 degrees from each other are equal and opposite as the two coils are physically wired together but with different winding directions in our design. The excitation current which tends to enhance the magnetic field of the actuator positioned at the positive direction of a steering axis is defined to be positive in the complete simulation model. The moving parts of the actuators that control the same steering motion move about the initial position $\left(L_{\mathrm{g}}=2\right.$ $\mathrm{mm})$ differentially. The motion which tends to increase the length of the air gap placed at the positive direction of a steering axis is defined to be positive.

The simulation results obtained from the complete model are shown by Table 1. From Table 1, it can be concluded that the force outputs of the two actuators that control one steering motion are not affected by the other two actuators which control the other steering motion. In other words, the force outputs of one pair of actuators are independent of those of the other pair of actuators for the complete model. Combing Table 1, Fig. 14 and Fig. 15, we can find that the force outputs from the simplified model are nearly the same as those from the complete model. Therefore, the force output of one actuator is

Table 1. Simulation results from the complete model.

\begin{tabular}{cccccccc}
\hline \hline $\begin{array}{c}\text { Current } \\
(\mathrm{X})\end{array}$ & $\begin{array}{c}\text { Current } \\
(\mathrm{Y})\end{array}$ & $\begin{array}{c}\text { Displacement } \\
(\mathrm{X})\end{array}$ & $\begin{array}{c}\text { Displacement } \\
(\mathrm{Y})\end{array}$ & $\begin{array}{c}\text { Force } \\
(-\mathrm{X})\end{array}$ & $\begin{array}{c}\text { Force } \\
(+\mathrm{X})\end{array}$ & $\begin{array}{c}\text { Force } \\
(-\mathrm{Y})\end{array}$ & $\begin{array}{c}\text { Force } \\
(+\mathrm{Y})\end{array}$ \\
\hline $0 \mathrm{~A}$ & $0 \mathrm{~A}$ & $0 \mathrm{~mm}$ & $0 \mathrm{~mm}$ & $1.36 \mathrm{~N}$ & $1.36 \mathrm{~N}$ & $1.36 \mathrm{~N}$ & $1.36 \mathrm{~N}$ \\
$0 \mathrm{~A}$ & $0 \mathrm{~A}$ & $0.4 \mathrm{~mm}$ & $0 \mathrm{~mm}$ & $2.02 \mathrm{~N}$ & $0.92 \mathrm{~N}$ & $1.36 \mathrm{~N}$ & $1.36 \mathrm{~N}$ \\
$0 \mathrm{~A}$ & $0 \mathrm{~A}$ & $0.4 \mathrm{~mm}$ & $0.4 \mathrm{~mm}$ & $2.02 \mathrm{~N}$ & $0.92 \mathrm{~N}$ & $2.02 \mathrm{~N}$ & $0.92 \mathrm{~N}$ \\
$3 \mathrm{~A}$ & $0 \mathrm{~A}$ & $0 \mathrm{~mm}$ & $0 \mathrm{~mm}$ & $0.79 \mathrm{~N}$ & $2.01 \mathrm{~N}$ & $1.36 \mathrm{~N}$ & $1.36 \mathrm{~N}$ \\
$3 \mathrm{~A}$ & $0 \mathrm{~A}$ & $0.4 \mathrm{~mm}$ & $0 \mathrm{~mm}$ & $1.31 \mathrm{~N}$ & $1.46 \mathrm{~N}$ & $1.36 \mathrm{~N}$ & $1.36 \mathrm{~N}$ \\
$3 \mathrm{~A}$ & $0 \mathrm{~A}$ & $0.4 \mathrm{~mm}$ & $0.4 \mathrm{~mm}$ & $1.31 \mathrm{~N}$ & $1.46 \mathrm{~N}$ & $2.02 \mathrm{~N}$ & $0.92 \mathrm{~N}$ \\
$3 \mathrm{~A}$ & $3 \mathrm{~A}$ & $0 \mathrm{~mm}$ & $0 \mathrm{~mm}$ & $0.79 \mathrm{~N}$ & $2.01 \mathrm{~N}$ & $0.79 \mathrm{~N}$ & $2.01 \mathrm{~N}$ \\
$3 \mathrm{~A}$ & $3 \mathrm{~A}$ & $0.4 \mathrm{~mm}$ & $0 \mathrm{~mm}$ & $1.31 \mathrm{~N}$ & $1.46 \mathrm{~N}$ & $0.79 \mathrm{~N}$ & $2.01 \mathrm{~N}$ \\
$3 \mathrm{~A}$ & $3 \mathrm{~A}$ & $0.4 \mathrm{~mm}$ & $0.4 \mathrm{~mm}$ & $1.31 \mathrm{~N}$ & $1.46 \mathrm{~N}$ & $1.31 \mathrm{~N}$ & $1.46 \mathrm{~N}$ \\
\hline
\end{tabular}




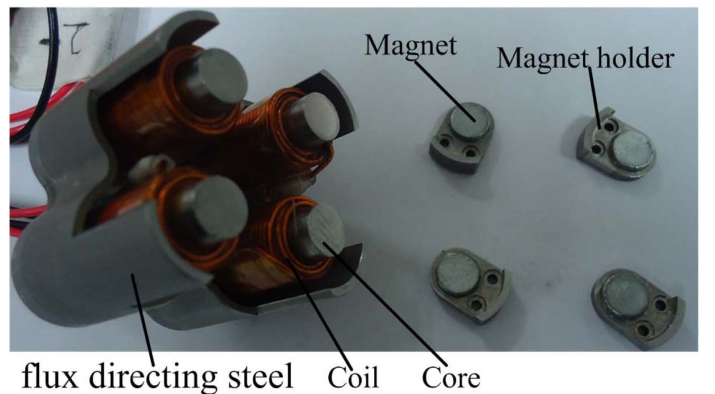

Fig. 12. (Color online) A photograph of the fabricated actuators.

independent of that of the other actuator for one pair of actuators. With these analysis results, the electromagnetic independence between each actuator is proved.

\section{Experimental Validation}

Based on the previous design results, each component of the actuators in the FSM is fabricated and is assembled together. Figure 12 is a photograph of the fabricated actuators. Note that the cores of the actuators are made to be a whole to simplify fabrication and assembling, and so do the flux directing steels.

In order to evaluate the force output of the fabricated actuator and validate the previous analysis results with experiment, an experimental test system is built. Figure 13 shows the components of the test system. As the coils controlling the same axis of steering motion are physically wired together, the coil positioned at 180 degrees from the tested actuator is also excited when a current is assigned to the coil of the tested actuator. However, the test is still effective because of the electromagnetic independence between each actuator. The precision electronic balance is used to measure the attraction force (force output) generated in the working air gap of the tested actuator. The micropositioner aims to adjust the air gap length.

Figure 14 and Fig. 15 shows the test force output within the designed range of the air gap length $\left(1.6 \mathrm{~mm} \leq L_{\mathrm{g}} \leq\right.$ $2.4 \mathrm{~mm}$ ), along with the simulation results. The test results in Fig. 14 confirm that the force output is nearly a linear function of the air gap length within the FSM travel range and Fig. 15 validates the linear dependency of the force output on the excitation current. The difference between the simulation results and test results is mainly caused by the residual magnetization of the core. Fabricating the

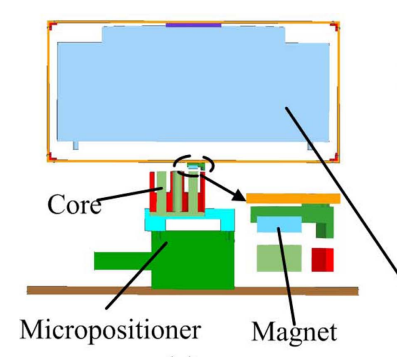

(a)

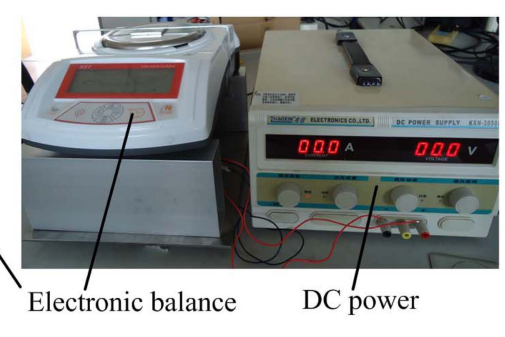

(b)

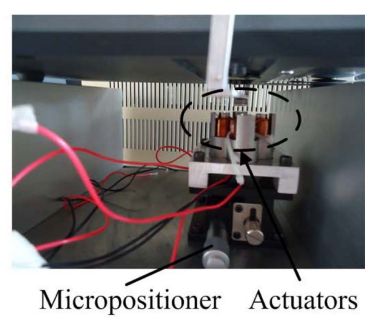

(c)

Fig. 13. (Color online) Experimental setup for evaluating force output. (a) Schematic cross-sectional view, (b) front view, (c) side view.

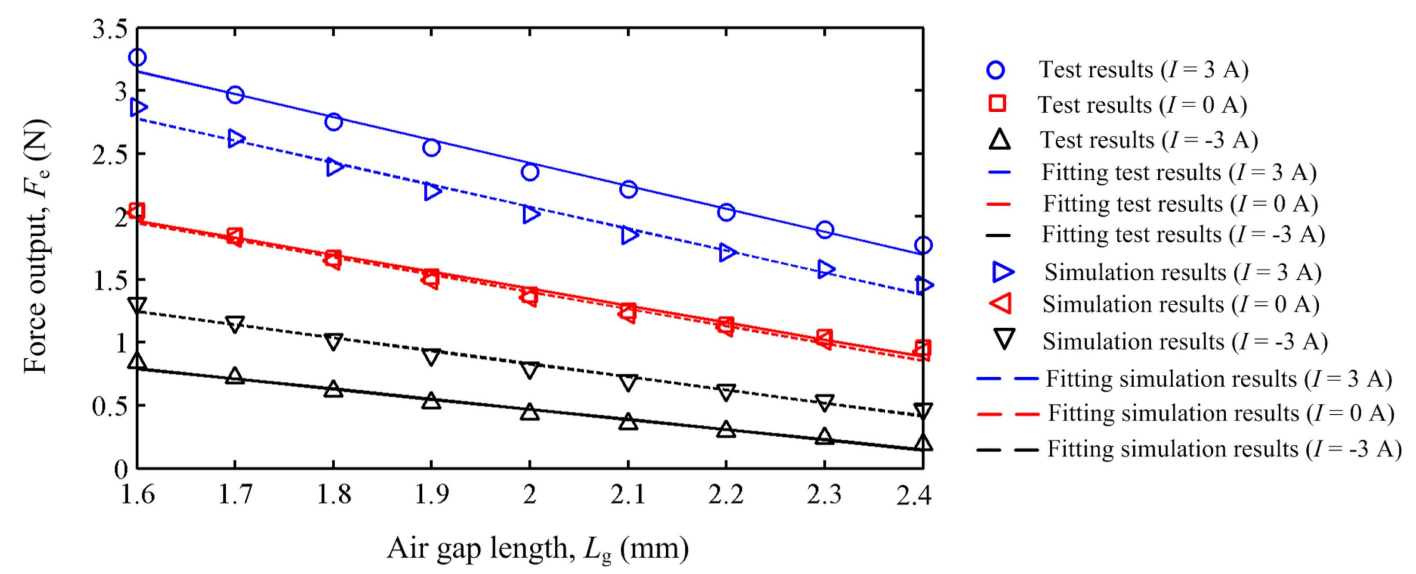

Fig. 14. (Color online) Variation of the simulation and test force outputs with the air gap length $\left(1.6 \mathrm{~mm} \leq L_{\mathrm{g}} \leq 2.4 \mathrm{~mm}\right)$. 


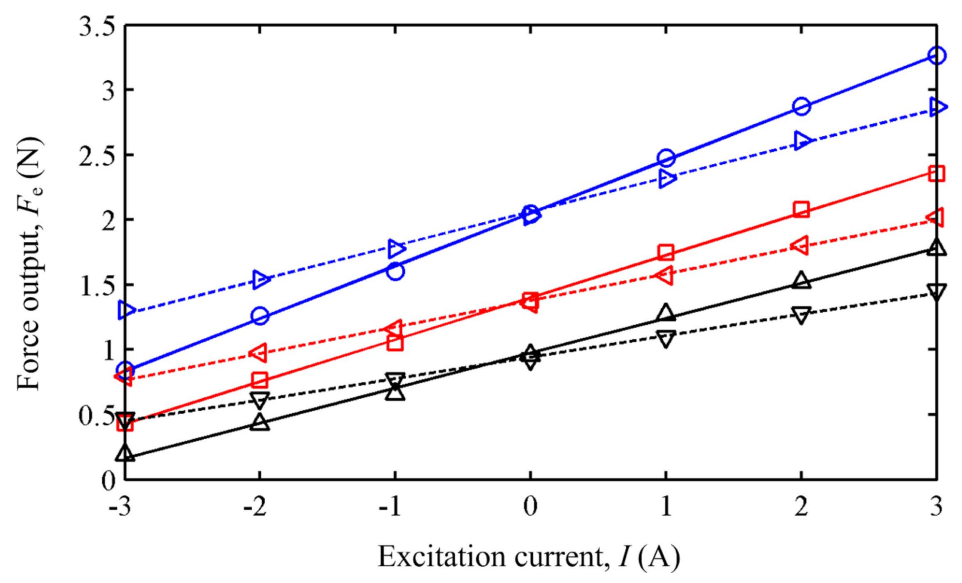

○ Test results $\left(L_{g}=1.6 \mathrm{~mm}\right)$

ㅁ Test results $\left(L_{g}=2.0 \mathrm{~mm}\right)$

$\Delta$ Test results $\left(L_{g}=2.4 \mathrm{~mm}\right)$

- Fitting test results $\left(L_{g}=1.6 \mathrm{~mm}\right)$

- Fitting test results $\left(L_{g}=2.0 \mathrm{~mm}\right)$

- Fitting test results $\left(L_{g}=2.4 \mathrm{~mm}\right)$

$\triangleright$ Simulation results $\left(L_{g}=1.6 \mathrm{~mm}\right)$

$\triangleleft$ Simulation results $\left(L_{g}=2.0 \mathrm{~mm}\right)$

$\nabla$ Simulation results $\left(L_{g}=2.4 \mathrm{~mm}\right)$

- - Fitting simulation results $\left(L_{g}=1.6 \mathrm{~mm}\right)$

- - Fitting simulation results $\left(L_{g}=2.0 \mathrm{~mm}\right)$

- Fitting simulation results $\left(L_{g}=2.4 \mathrm{~mm}\right)$

Fig. 15. (Color online) Variation of the simulation and test force outputs with the excitation current $\left(1.6 \mathrm{~mm} \leq L_{\mathrm{g}} \leq 2.4 \mathrm{~mm}\right)$.

core with material of low residual magnetization and small coercive force, such as pure iron, is able to decrease the deviation between the simulation results and the experiment results. But for quick concept verification, it is preferred to use stainless steel due to its high saturation induction, relative good machinability and the current availability in our laboratory. In fact, the effect of the residual magnetization can be compensated with control algorithm. In addition, fabrication errors of the cores and permanent magnets also contribute to the difference between the simulation results and test results, especially the magnetization curve of the core material.

\section{Conclusion}

This paper presents a moving-magnet actuator for fast steering mirror. The actuator enables a reasonable compromise between voice coil actuator and piezoelectric actuator and has a linear dependency on air gap length and excitation current. Both the magnetic analysis and optimal design of the actuator are implemented through finite element simulation. Based on the designed results, a prototype of the actuator is fabricated. An experimental test system is then built to examine its force output. The experiment results validate the actuator analysis and design.

\section{Acknowledgements}

This project is supported by National Natural Science
Foundation of China (Grant No. 51375303).

\section{References}

[1] G. C. Loney, Proc. SPIE 1454, 198 (1991).

[2] M. Hafe, T. C. Sidler, R. P. Salathé, G. L. M. Jansen, and J. C. Compter, Mechatronics 10, 741 (2000).

[3] K. Aoki, Y. Yanagita, H. Kuroda, and K. Shiratama, Proc. SPIE 5160, 495 (2004).

[4] X. Wu, S. Chen, B. Shi, W. Chen, and X. Xiong, Opt. Eng. 50, 023002 (2011).

[5] Y. Lu, D. Fan, and Z. Zhang, Optik 124, 2443 (2013).

[6] H. Marth and M. Donat, Proc. SPIE 1543, 248 (1991).

[7] C. T. Wood, G. C. Homer, and W. W. Clark, Proc. SPIE 3674, 196 (1999).

[8] S. Woody, and S. Smith, Mechatronics 16, 389 (2006).

[9] S. Bing, R. Weibin, G. Bin, R. Changhai, and S. Lining, Smart Mater. Struct. 17, 025032 (2008).

[10] S. Bing, C. Liguo, R. Weibin, R. Changhai, and X. Min, Smart Mater. Struct. 18, 035009 (2009).

[11] P. Bandera, Proc. 8th European Space Mechanisms and Tribology Symposium ESA, SP-438 (1999).

[12] D. J. Kluk, M. T. Boulet, and D. L. Trumper, Mechatronics 22, 257 (2009).

[13] X. Lu, Ph.D. Thesis, Massachusetts Institute of Technology, America (2005).

[14] D. Wu, X. Xie, and S. Zhou, IEEE Trans. Magn. 46, 1007 (2010). 